\title{
CHRISTOPH SCHUBERT / ERLANGEN
}

\section{Textkritisches zu Augustins De Genesi ad litteram (Fragmentum Latinum Jenense 48)}

Die textkritische Arbeit an Augustins großer Genesis-Auslegung ist seit der Edition von Joseph Zycha ${ }^{1}$ nicht wesentlich vorangekommen. Verstreut finden sich in der Literatur Verbesserungsvorschläge zu einzelnen Stellen, wichtige Hinweise bei Taylor ${ }^{2}$ und in der zweisprachigen Ausgabe von Agaësse und Solignac, die Zychas Text im wesentlichen übernimmt. ${ }^{3}$

Zycha stützte sich lediglich auf die fünf ältesten ihm bekannten Handschriften aus dem 7. bis 9. Jh.: einen Codex Sessorianus $(E)$, zwei Parisini $(P$ und $R$ ), einen Sangallensis $(S)$ und einen Berolinensis $(B) .{ }^{4}$ Ergänzend zog er

\footnotetext{
${ }^{1}$ Sancti Aureli Augustini De Genesi ad litteram libri duodecim ... recensuit Iosephus Zycha, Prag-Wien-Leipzig 1894 (CSEL XXVIII 3,1), nachgedruckt Wien 1970. Zychas Edition erfuhr reichlich Kritik, vgl. A. Solignac in: CEuvres de Saint Augustin. La genèse au sens littéral en douze livres. Traduction, introduction et notes par P. Agaësse et A. Solignac (= BAug 48/49), 2 Bde., Paris 1972, nachgedruckt Paris 2000, 55 - 61 mit abgewogenem Urteil.

2 J. H. Taylor, The Text of Augustine's De Genesi ad Litteram, Speculum 25 (1950), 87 - 93, hat drei von Zycha nicht berücksichtigte jüngere Handschriften zum 12. Buch vollständig kollationiert und teilt daraus 89-92 eine Reihe bedenkenswerter Lesarten mit. Hinweise auf fünf weitere jüngere Handschriften 92. Für seine Übersetzung: St. Augustine, The literal meaning of Genesis, Translated and annotated by J. H. Taylor, 2 Bde., New York u. a. 1982, hat Taylor zusätzlich zu Zychas Handschriften acht weitere fallweise eingesehen und teilt daraus in den Anmerkungen einzelne Lesarten mit; vgl. zur Vorgehensweise mit einer Liste der Manuskripte I 14f.

${ }^{3} \mathrm{Zu}$ den Prinzipien ihrer Edition vgl. Solignac (o. Anm. 1), $63 \mathrm{f}$.

${ }^{4}$ Es sind dies nach Zycha (o. Anm. 1), praef. VI-XI, der „Codex olim Sessorianus membranaceus n. XIII, ... nunc numero 2094 signatus in bibliotheca Nationali Vict. Emman. ..., s. VII., ... olim in bibliotheca Santa Croce“ (E); der „Codex Parisinus n. 2706 olim Colbertinus 5150, tum Regius 400.2 membranaceus s. VII-VIII“ $(P)$; der „Codex olim Colbertinus 894, tum Regius 3538 a 12, nunc Parisinus n. 1804, membranaceus s. VIIII“ $(R)$; der „Codex Sangallensis n. 161 membranaceus (+ 2 folia papyracea) s. VIIII“ $(S)$ und der „Codex Berolinensis n. 24 ... ex bibl. Meerman. cod. Phillip. 1651, membranaceus s. VIIII - X“ $(B)$. Die Lesarten des Berolinensis, der Zycha zu spät zugänglich wurde, finden sich nicht im Apparat der Ausgabe, sondern als notabiliora in der praefatio zur Ausgabe XI-XIIII zusammengestellt. Zychas Leithandschrift, der in sorgfältiger Un-
} 
die Baseler editio princeps von 1506 (b) und die Ausgabe der Mauriner (d), die Excerpta Eugippii in der Edition Knoells von 1886 und - dies nur fallweise - eine Kölner Handschrift des 12. Jh. (C) heran. ${ }^{5} \mathrm{Zu}$ den jüngeren Handschriften des im Mittelalter beliebten und weit verbreiteten Textes bemerkte er lapidar: „saeculum duodecimum et proxime sequentia libris manu scriptis abundant". 6

Die folgenden Mitteilungen zu einem bisher nicht bestimmten und jüngst erst signierten Fragment, das in der Handschriftenabteilung der Universitätsbibliothek Jena aufbewahrt wird, wollen zeigen, daß die Berücksichtigung der recentiores durchaus Fortschritte bei der Textkonstitution erwarten läßt. Vorangestellt wird eine Beschreibung des Fragments.

Das Jenenser Fragment besteht aus einem einspaltig beschriebenen, an allen vier Seiten beschnittenen Doppelblatt kräftigen gelblichen Pergaments, dessen ursprünglicher Mittelfalz und Bindungsspuren noch deutlich erkennbar sind. Die Maße des heute auseinandergefalteten Blattes betragen $372 \mathrm{x}$ $245 \mathrm{~mm}$. Aus der Lage des Mittelfalzes ergibt sich mindestens eine Seitenbreite von $215 \mathrm{~mm}$. Die Seitenhöhe läßt sich aufgrund der starken Beschneidung des oberen Randes nicht sicher bestimmen, muß bei gleichmäßiger Textverteilung aber etwa $350 \mathrm{~mm}$ betragen haben. Der Zeilenspiegel dürfte bei ursprünglich 30 oder 31 Zeilen (erhalten sind maximal 25) $140 \mathrm{x}$ ca. 240 $\mathrm{mm}$ gemessen haben. Zirkellöcher für die noch gut sichtbare einfache Linierung sind am linken Rand der heutigen Vorderseite (Fleischseite), die die aktuelle Signatur trägt, zu sehen.

Das Blatt diente als Einband des vom Darmstädter Hofprediger Henricus Leuchterus besorgten und von Veit Hase gedruckten Bandes Antiqua Hessorum fides christiana et vera: das ist, Historischer Bericht, vom alten und

ziale geschriebene Sessorianus, wird heute schon ins 6. Jh. datiert, vgl. Taylor 1950 (o. Anm. 2), 87 mit Anm. 1. Die verschiedenen Hände der Handschriften $\left(R^{1}, E^{3}\right.$ etc.) werden im folgenden nach Zycha angegeben.

5 „Codex Coloniensis n. 61 s. XII, membranaceus miscell.“, eine Beschreibung bei Zycha (o. Anm. 1), praef. X, der die Nähe zu $S$ betont. Da $C$ nur unvollständig kollationiert und im Apparat nachgewiesen ist, bleibt er für die textkritischen Überlegungen im folgenden ohne Berücksichtigung.

${ }^{6}$ Zycha (o. Anm. 1), praef. VI. Die noch nicht abgeschlossene Sichtung der Bestände bestätigt Zychas Ansicht. In der 1969 begonnenen Reihe „Die handschriftliche Überlieferung der Werke des Heiligen Augustinus“, die von der Kirchenväterkommission der Österreichischen Akademie der Wissenschaften betreut wird, sind außerdem etliche von Zycha nicht berücksichtigte Handschriften aus dem 8. und 9. Jh. nachgewiesen. Für den Bereich der alten Bundesrepublik belegt Rainer Kurz im 1976 erschienenen 5. Band der Reihe sechs Handschriften des 12. Jh. (123). Das bei Zycha angedeutete und von Solignac (o. Anm. 1), 61 -63 klarer skizzierte Stemma der von Zycha zugrundegelegten Handschriften dürfte als Grundgerüst einer künftigen Edition nur bedingt geeignet sein. 
wahren Christlichen Glauben oder Religion der Hessen, Darmstadt 1607, wobei die heutige Vorderseite die Außenseite bildete. An allen vier Seiten war ein Streifen Pergament eingeschlagen. Der zentrale Bereich des ehemaligen Buchrückens ist mit Absicht stark berieben und trägt - heute kaum mehr lesbar - am unteren Rand den Titel der Antiqua Hessorum fides. Links oben und unten findet sich deren Signatur „Hist. eccl. V. q. 13“. Die Faltungsspuren erlauben die Rekonstruktion des alten Buchblocks mit etwa 195 x 155 x $35 \mathrm{~mm}$. Im Bereich der Faltungen und des Buchrückens ist der Text, der im übrigen gut lesbar ist, zerstört; Textverlust ist außerdem durch die Beschneidungen am oberen und in geringem Umfang auch am rechten Rand eingetreten. Durch eine ältere Restaurierung wurde der rechte Rand verstärkt.

Der Text aus Augustins De Genesi ad litteram setzt auf der heutigen Rückseite auf $1^{\mathrm{r}}$ im 9. Buch ein und umfaßt auf der ersten Seite fast das gesamte sechste Kapitel, von hac vita oportebat ..., sicher lesbar erst ab de]cessiones et successiones ... $\left(273,24^{7}\right)$ bis probabilius primis illis $(275$, 4). Auf $1^{\mathrm{v}}$ folgt aus dem siebten und achten Kapitel der Text von nihilominus quare subtrahatur ..., sicher lesbar erst ab ho]noris meritum apud deum ... $(275,13)$ bis eorum contraria perniciter $(276,15)$, worauf in derselben Zeile wohl noch currant etenim folgte. $2^{\mathrm{r}}$ beginnt im 19. Kapitel mit uxo]ri suae et erunt ... (294, 11) und fährt nach kurzem Zwischentitel (Explicit liber nonus. Incipit liber decimus) mit dem Anfang des 10. Buches bis etiam de anima viri $(295,19)$ fort. $2^{\mathrm{v}}$ beginnt im ersten Kapitel mit propterea putant, sicher lesbar ab animam mulieris ex anima ... $(296,2)$ und endet im zweiten Kapitel mit dictum est: mascu[lum (297, 4f.). Aufgrund der Textverteilung dürften sich ursprünglich drei weitere Doppelblätter zwischen den beiden Hälften des Fragments befunden haben.

Der in schwarzbrauner Tinte geschriebene Text dürfte von einem einzigen Schreiber stammen. Die Schrift ist eine aufrechte, etwas steife, aber sehr regelmäßige und sorgfältig ausgeführte Minuskel, die in das 12. Jh. weist. Buchstabenformen und Kürzungen sind mit erstaunlicher Konsequenz verwendet. Doppelformen begegnen überhaupt nicht: als $a$ dient stets ein aufrechtes kleines unziales $a$ mit häkchenförmigem, teils nur angedeutetem Anstrich; als $d$ das für die Gotik charakteristische runde $d$ mit geradem Ansatz der Oberlänge ; für ae tritt stets, auch in Kürzungen, $e$ caudata ein; das runde $s$ begegnet neben dem gewöhnlichen, auf der Zeile stehenden geraden ausschließlich in der Majuskelligatur US. Charakteristisch für die Schrift ist die Tendenz, die Buchstaben, etwa $f$ und $s$, aber auch den Bogenschluß von $e$ oder $g$ mit Haarstrich auslaufen zu lassen, teils auch Haarstriche als Zierstrich anzufügen und viele Buchstaben mit kurzem Haarstrich anzusetzen. Die Nei-

\footnotetext{
${ }^{7}$ Die folgenden Seiten- und Zeilenangaben stets nach der Ausgabe Zychas.
} 
gung zu eckiger Gestaltung der Buchstaben ist bei $a, b$ und $h$ besonders ausgeprägt, Brechungen begegnen aber nicht. Charakteristisch ist auch der häufige Einsatz von Großbuchstaben am Satzanfang. Bei diesen ist die Verdikkung der Senkrechten und der Versuch, quadratische Proportionen zu erreichen, auffällig. Das $g$ wird wie doppeltes $c c$ angesetzt; der zweite Schwung, der einen großen Bauch öffnet und in einer kräftigen Waagrechten endet, wird teils mit einem neu angesetzten Haarstrich nach oben gezogen, teils bleibt der Bauch offen. Nur über doppeltem $i i$ sind i-Striche gesetzt. Schlecht fügt sich in das ansonsten harmonische Schriftbild das $y$ (ähnlich der USLigatur) ein.

Als Ligaturen sind nur US, st und $c t$ vorhanden. Das gewöhnliche Kürzungszeichen ist der waagrechte Strich, der, wo immer es möglich ist, an den vorausgehenden Langbuchstaben angehängt wird. Er steht für $n / m$, aber auch für verschiedene Suspensionskürzungen. ${ }^{8}$ Daneben werden nur die 2-förmige $u r$-Kürzung, die 9-förmige $u s$-Kürzung sowie übergeschriebene Buchstaben verwendet. Die Kürzungen sind ansonsten die üblichen, ${ }^{9}$ auffällig nur die konsequente Kürzung aller Formen von habere $(h+$ Endung). Zur Interpunktion wird der Punkt und das Fragezeichen verwendet, dieses in Form einer halb geschlossenen liegenden 8 , teils auch nur als Tilde über dem Punkt.

In hellroter Tinte sind Explicit und Incipit und die schlicht, aber geschmackvoll mit gefüllten Voluten und Halbkreisen als Außenmotiven gestaltete, $60 \mathrm{~mm}$ hohe Zierinitiale (I von Iam) zu Beginn des 10. Buches gehalten, $a$ und $m$ mit roten Punkten geschmückt. Als Auszeichnungsschrift ist für Explicit und Incipit eine mit Minuskelbuchstaben durchsetzte, der Unziale nahestehende Majuskel verwendet. Nur hier findet sich unter den Minuskelbuchstaben das kleine gerade $d$.

Lediglich zwei kurze, durch die Beschneidung unvollständige Marginalglossen begegnen auf $1^{\mathrm{v}}$ oben und in der Mitte: ... virginitas obser / ... erus (?) und ... ptus exposit', darüber eine NT-Ligatur, mit starker Verlängerung des zweiten Schaftes, welche durch zwei kleine Kreise oben und unten abgesetzt ist. Tinte und Schrift sind die des Textes.

Der Vergleich der Lesarten mit den bei Zycha nachgewiesenen ergibt ein verhältnismäßig klares Bild. ${ }^{10}$ Das Jenaer Fragment steht deutlich dem

${ }^{8}$ Über dix für dixit, er für erunt, nich für nichil, parit für pariter, sic für sicut.

${ }^{9}$ So für die nomina sacra, für die Präfixe per-, pri-, pro-, für kleine Wörter wie non $(n)$, -que (q;), quod (qd), quando (qn), quoniam (qm), sed (s;), sunt (s), tamen (tn), vel (ul und $l$ ) etc. Als Kürzung für et dient der tironische $e t$-Haken, derselbe überstrichen für etiam.

${ }^{10}$ Die nicht allzu zahlreichen orthographischen Varianten bleiben im folgenden unberücksichtigt. 
Sangallensis $(S)$ am nächsten. Mit diesem allein teilt es folgende Fehler resp. Varianten:

- Umstellungen: sunt accepturi pariter (274,9f.); similiter flando (295, 17); nascentium hominum $(296,5)$; putaremus factum $(296,14)$; mulieri esse $(296,18){ }^{11}$

- Auslassungen: enim $(274,14)$; factum $(296,7)$; eius $(296,26)$.

- andere Abweichungen: replesse statt prolis $(275,17) ;{ }^{12}$ incontinenter statt incontinentes $(275,22) ;{ }^{13}$ autem statt namque $(276,14)$; dimittit statt dimittet $(294,13)$; primum statt primo $(295,13)$; finiri terminus statt terminus $(294,19 \mathrm{f}.){ }^{14}$ enim statt autem $(295,4)$; animantiusque statt amantiusque $(296,21){ }^{15}$

Hinzu treten die Fehler bzw. Varianten, die das Fragment und $S$ mit weiteren Handschriften gemeinsam haben:

- Umstellung: necessitudo non postulat mit PRSbd $(275,17 \mathrm{f}.) .{ }^{16}$

- andere Abweichungen: quod mit EPRb statt quo $(275,25) ;{ }^{17}$ propter mit PRSCbd statt per $(294,18){ }^{18}$ dictum mit $R^{l} S C$ statt ductum $(295$, 17); tunc mit PRSd(b) statt tum (297,1); quemadmodum mit $S$ (quemammodum E) statt quem modum (297,2f.). ${ }^{19}$

\footnotetext{
${ }^{11}$ Nur mulieri und anschließende Lücke im Fragment.

${ }^{12}$ Nur repl lesbar.

${ }^{13}$ Mit incontinentes steht die Phrase conubium vel quo incontinentes copulantur in oratorischer Fülle für conubium vel incontinentium und deutet die in der Ehe anhaltende incontinentia nur an. Mit incontinenter wäre diese drastischer ausgedrückt, copulari dann nicht für die Verbindung durch das Band der Ehe, sondern für die körperliche Vereinigung gesetzt. Zu dem ruhigen Duktus der Passage paßt incontinentes wohl besser.

${ }^{14}$ Pleonasmen beim Ausdruck des Beginnens und Aufhörens sind in der lateinischen Literatur häufig, vgl. E. Löfstedt, Syntactica. Studien und Beiträge zur historischen Syntax des Lateins, Zweiter Teil. Syntaktisch-stilistische Gesichtspunkte und Probleme, Lund 1956, 173f. und 179f. Die Variante ist bedenkenswert.

${ }^{15} \mathrm{Da}$ anima mehrmals vorausgeht, wird man in animantiusque zunächst eine Verschreibung sehen. Indes scheint auch ein Wortspiel mit anima denkbar; dann wäre animantiusque original.

${ }^{16}$ Der Prosarhythmus spricht für den Doppelkretiker necessi]tudo non postulat (mit geläufiger Endsilbenkürzung bei necessitudo); bei non postulat necessitudo wäre der Ditrochäus mit vorausgehender Kürze im fünfsilbigen Wort ungebräuchlich.

${ }^{17}$ Mit quod wären dieser und der vorausgehende quod-Satz nicht relativisch, sondern faktisch aufzufassen.

${ }^{18}$ propter dürfte nach Ansicht Zychas von den vorausgehenden Bibelstellen, in denen zweimal propter vorkommt, induziert worden sein. per ist allerdings wegen des Plusquamperfekts in praecesserat mißlich (die extasis müßte in der Phrase per extasin dicere an sich als noch andauernd gedacht sein). Daher ist propter vielleicht vorzuziehen.

${ }^{19}$ Vielleicht original. tenere wäre dann absolut gebraucht, die Nebensätze mit quaenam und quemadmodum leicht inkonzinn formuliert - wohl die lectio difficilior.
} 
Der exakte Zusammenhang zwischen $S$ und dem Fragment ist nicht leicht zu ermitteln, da das Fragment offenbar von einem mitdenkenden Schreiber verfaßt wurde, so daß spontane Korrekturen nicht auszuschließen sind. ${ }^{20}$ Plausibel scheint ein naher gemeinsamer Vorfahre, vielleicht die Vorlage von $S$, wobei zwischen diesem Vorfahren und dem Fragment allein schon aufgrund der zeitlichen Abfolge eine Stufe mehr als zwischen ihm und $S$ liegen dürfte. Sollte Kontamination vorliegen, ist nicht auszuschließen, daß das Fragment zu einem codex descriptus, der dann auf $S$ zurückginge, gehörte, freilich aus guter Quelle kontaminiert wurde.

Wenige Sonderfehler des Fragments gegen $S$ und die übrige Überlieferung sind zu verzeichnen: et statt ad $(275,15)$; cum altera vel cum altero statt cum altera vel altero $(276,1)$; primum statt primam $(296,6) ;{ }^{21}$ unam statt una $(296,7)$. Außerdem zwei Umstellungen: fine saeculi $(274,4)$ und tamen ille $(274,11)$. Hinzu kommen veniabile mit $E^{3}$ statt veniale (275, $24)^{22}$ und testatur mit $B$ statt testetur $(294,13)$.

Sonderfehler von $S$ gegen das Fragment und die übrige Überlieferung: omne statt omnem $(276,13)$; ipse $S^{1}$ statt ipsa $(294,13)$; die Umstellung animam esse factam $(296,15)$. Außerdem primum mit PRbd statt primo $(296,25)$.

Besonders charakteristisch für den Zusammenhang scheinen mir folgende Stellen:

- suscipiatur - nutriatur - educetur $(276,1 \mathrm{f}$.) in der übrigen Überlieferung; suscipiantur - nutriantur - edocentur S; suscipiantur - nutriantur - edoceantur im Fragment, das das grammatikalisch falsche edocentur, das in der Vorlage gestanden haben wird, sekundär korrigiert.

- inquiramus $(295,6)$ die übrige Überlieferung; disquiramus $S$; disseramus das Fragment, als Augensprung zu 295, 3 zu erklären, der gut von einem disquiramus, schwerlich von inquiramus ausgelöst sein kann.

${ }^{20}$ An einer einzigen Stelle - et statt ad $(275,15)$ - ist eine sinnvolle Auflösung nicht möglich. Sonst ergeben alle, auch die klar sekundären Varianten, stets einen grammatikalisch wie inhaltlich sinnvollen Text.

${ }^{21}$ Hier liegt wohl eine Verschreibung vor, die sich aus dem fälschlichen Bezug von primum auf das unmittelbar vorausgehende hominum erklärt. Indes ließe sich der Satz auch mit primum sowohl als temporalem Adverbiale als auch als maskulinem Akkusativ (zu ergänzen: hominem) verstehen, beides im Sinne von: „wie Gott bei der Erschaffung des ersten Menschen handelte."

${ }^{22}$ Das gleichbedeutende veniabile gibt den deutlicheren Gleichgang mit dem vorausgehenden culpabile $(275,23)$ und wurde wohl dadurch ausgelöst. Augustin kennt veniabilis (veniabiliora in epist. 153 vol. 44 par. 1397,10 und 15), bevorzugt aber klar veniale, auch in Kombination mit anderen Adjektiven auf -bilis. 
- prophetam die übrige Überlieferung $(294,19)$; propheta $S$ prophetam das Fragment mit expungiertem $-m$, das keinen absolut sicheren Schluß zuläßt, aber auf eine Vorlage mit propheta weist.

Die Qualität der Überlieferung des Jenaer Fragments zeigt sich in der insgesamt geringen Zahl der Fehler, einigen bedenkenswerten Varianten ${ }^{23}$ besonders deutlich aber an der folgenden Stelle, die allein den ursprünglichen Text bewahrt haben dürfte. In De Genesi 9, 6 bietet die übrige Überlieferung übereinstimmend:

Neque enim arbitrandum est Heliam vel sic esse iam, sicut erunt sancti, quando peracto operis die denarium pariter accepturi sunt, vel sic, quemadmodum sunt homines, qui ex ista vita nondum emigrarunt. de qua ille tamen non morte, sed translatione migravit. iam itaque aliquid melius habet, quam in hac vita posset, quamvis nondum habeat, quod ex hac vita recte gesta in fine habiturus est $(274,8-14)$.

Der Passus gehört zu einem Argumentationsgang, in dem Augustin nachzuweisen versucht, daß die Bestimmung der Frau von Anfang an in der Mithilfe bei der generatio filiorum lag und ihr nicht erst sekundär nach dem Sündenfall zukam, der die Lebenszeit durch den Tod begrenzte und so zum Fortbestand der Menschheit Zeugung und Geburt zwingend notwendig machte. In diesem Zusammenhang wird die theoretische Möglichkeit einer Fortpflanzung noch im paradiesischen Urzustand verteidigt und - um eine Übervölkerung des Gartens Eden zu vermeiden - anstelle des Todes die translatio in einen nicht-körperlichen Zustand erwogen. Als historischer Beweis für die Möglichkeit eines solchen Übergangsstadiums von bereits rein geistig-seelischem Sein vor der völligen Erlösung am Jüngsten Tag werden Elia und Enoch angeführt. Elia, so unser Abschnitt, schwebt zwischen der Körperlichkeit dieser Welt und der endgültigen Verklärung. ${ }^{24}$ Der Text legt in der zitierten Form mit itaque Nachdruck auf die translatio (im Gegensatz zur mors), also auf die Form des Übergangs, die für den aus ihr folgenden Zwischenzustand entscheidende Bedeutung besitzt.

Das Fragment bietet hingegen: ... de qua tamen ille non morte, sed translatione migravit, iam utique aliquid melius habens,

${ }^{23}$ Vgl. o. die Anm. 14 bis 19.

${ }^{24}$ Die Auffassung, daß die Heiligen erst am Jüngsten Tag mit allen anderen Menschen in der zweiten Auferstehung zur vollen Gegenwart Gottes gelangen, teilt Augustin mit der ganzen Alten Kirche, vgl. A. Angenendt, Heilige und Reliquien, München 1994, 102-106 und ders., Geschichte der Religiosität im Mittelalter, Darmstadt ${ }^{2} 2000$, 684-689 sowie R. Staats, Art. Auferstehung I/4, TRE 4,477. Hier wird sie mit der Anspielung auf das Gleichnis von den Arbeitern im Weinberg (Matth. 20, 1-16) biblisch fundiert. Zum Inhalt des Passus vgl. auch Taylor 1980 (o. Anm. 2), II 265 f. 
quam in hac vita possit, quamvis nondum habeat, quod ex hac vita recte gesta in fine habiturus est.

Mit utique rückt die Form des Übergangs zur Seligkeit in den Hintergrund. Betont wird die Mitte, die Elias Zustand zwischen Körperlichkeit und völliger Verklärung hält, und die Schwierigkeit, die seine genaue Bestimmung macht. Da eben dieser schwer definierbare mittlere Zustand Thema des Abschnitts ist, wird man utique und habens, das als Partizip besonders geeignet ist, die Dauerhaftigkeit der neuen Daseinsform auszudrücken, wohl vorziehen. Paläographisch ist der Übergang von utique zu itaque und von habens zu habet wie auch in umgekehrter Richtung unproblematisch, wobei itaque im folgenden eine finite Verbform nahelegt und vielleicht nach sich gezogen hat. ${ }^{25}$ Die Junkturen iam itaque / iam utique und itaque aliquid / utique aliquid sind allesamt gut augustinisch. ${ }^{26}$ Zwischen possit und posset wird sich schwer entscheiden lassen. Stilistisch erhält der Satz mit dem Partizip habens größeren Schwung und Eleganz; etwas wuchtiger und kurzatmiger, zumal mit itaque, ist das finite habet. So dürfte der originale Text wie im Fragment gelautet haben. Wie und wann es zum Eindringen des Fehlers in den Hauptstrang der Überlieferung kam, ließe sich sinnvoll erst auf der Grundlage einer editio maior beantworten.

Abschließend bleibt nur, Taylors Mahnung zu wiederholen, die seit einem halben Jahrhundert nichts von ihrer Gültigkeit eingebüßt hat: "It seems, then, that there is a genuine need for a new critical edition of the $D e$ Genesi ad Litteram, and that in the preparation of a new text the later manuscripts cannot be completely ignored. ... Until at least some of all these manuscripts neglected by Zycha are examined, we will not have a reliable critical text." 27

${ }^{25}$ In den 25 Belegen für iam itaque in den echten Schriften Augustins (nach dem Suchergebnis in CLCLT-4, release 2000) bezieht sich iam itaque ein einziges Mal nicht direkt auf das Prädikat des Hauptsatzes: Contra Iulianum opus imperfectum 6,26 (PL 41, 1564, 52f.).

${ }^{26}$ Nach den Suchergebnissen in CLCLT - 4 gebraucht Augustin itaque mit einer Form von aliquid achtmal, utique und aliquid sechsmal; iam itaque begegnet an 25 Stellen, iam utique an 22. Zum Gebrauch eines Vergangenheitstempus mit dem Partizip habens vgl. am ähnlichsten serm. 36, 11 (CC. SL 41 ed. C. Lambot 443, 286): pauperem se ille cognovit iam aliquid habens.

27 Taylor 1950 (o. Anm. 2), 92. Allen Mitarbeitern der Handschriftenabteilung der Thüringer Universitäts- und Landesbibliothek Jena sei schließlich für die stets freundliche Hilfe bei der Arbeit an den Jenenser Fragmenten gedankt, Frau Prof. Huber-Rebenich, Jena, und der Redaktion der Wiener Studien für wichtige Hinweise zur Beurteilung der Textvarianten. 